\title{
Simulation of organic interstellar dust in the laboratory
}

\author{
Walt W. Duley \\ Department of Physics and Astronomy, University of Waterloo, \\ 200 University Ave. West, Waterloo, Ontario, Canada N2L3G1 \\ email: wwduley@uwaterloo.ca
}

\begin{abstract}
New techniques for the generation and analysis of carbon nanoparticles (CNPs) have been developed and have resulted in the production of CNP samples whose infrared spectral properties are essentially identical to those observed in interstellar absorption and emission. These particles are of mixed aromatic/aliphatic composition. Spectra of CNPs containing $10^{2}-$ $10^{4}$ atoms will be discussed in relation to spectra of interstellar materials. We find that infrared line widths in these samples are typically $10-30 \mathrm{~cm}^{-1}$, but can be as small as $2 \mathrm{~cm}^{-1}$. Simulation of the $3.28 \mu \mathrm{m}$ feature is shown to yield important new insight into the nature of interstellar CNPs.
\end{abstract}

Keywords. Infrared: ISM, ISM: molecules, ISM: lines and bands, (stars:) circumstellar matter

\section{Introduction}

What is "organic interstellar dust"? Although one tends to think of a particular material, the composition of "organic interstellar dust" is undoubtedly not unique as the observed properties of this material vary depending on location in the ISM. In the diffuse interstellar medium (DISM), organic dust appears to have a composition similar to that of hydrogenated amorphous carbon (HAC), while dust seen in emission seems to consist of carbon nanoparticles (CNPs) having mixed polycyclic aromatic hydrocarbon (PAH) and aliphatic composition. The morphology of these materials is indicated to be that of aggregates of CNPs forming larger particles with an enhanced aliphatic composition in the DISM, while material in emission objects consists of individual CNPs having fewer aliphatic chains. In this model, the aliphatic chains act as "glue" between aromatic components in CNPs. A hierarchical model in which HAC is decomposed in the vicinity of emission objects is indicated by observation.

Key information on the composition of organic interstellar material can be obtained from infrared spectra of many circumstellar, interstellar and extra-galactic sources as these are dominated by emission from PAHs (Allamandola et al. 1989, Peeters et al. 2002, 2004, van Diedenhoven et al. 2004, Sloan et al. 2005) The strongest emission features (historically called the unidentified infrared (UIR) bands) occur at 3.3, 3.4, 6.2, 7.7, 8.6, 11.3 and $12.7 \mu \mathrm{m}$ and have been known for many years, but recent observations indicate that there are numerous other weaker bands (Werner et al. 2004, Sloan et al. 2005). Observations have shown that there are three basic types of emission spectra as indicated by the relative intensity and characteristic profile of features in the $3.3 / 3.4,6-9$ and 11-11.4 $\mu \mathrm{m}$ wavelength range (Geballe 1997, Tokunaga 1996, Peeters et al. 2002, van Diedenhoven et al. 2004). Adopting the notation of Peeters, most sources can be classified as type A, with a pronounced feature at 6.19-6.23 $\mu \mathrm{m}\left(1616-1605 \mathrm{~cm}^{-1}\right)$ together with bands at $\sim 7.6 / 7.8 \mu \mathrm{m}\left(1316 / 1280 \mathrm{~cm}^{-1}\right)$ and $\sim 8.6 \mu \mathrm{m}\left(1163 \mathrm{~cm}^{-1}\right)$. Type B sources have bands at 6.24-6.28, 7.6/7.9 and $8.7 \mu \mathrm{m}\left(1603 / 1592,1316 / 1265\right.$ and $\left.1150 \mathrm{~cm}^{-1}\right)$. 
A few type $\mathrm{C}$ objects (e.g., IRS 13416) exhibit features at $\sim 6.3$ and $8.2 \mu \mathrm{m}$ (1587 and $\left.1220 \mathrm{~cm}^{-1}\right)$ but do not have a band near $7.7 \mu \mathrm{m}\left(1300 \mathrm{~cm}^{-1}\right)$. In practice, some sources combine type A and type B characteristics, and the observed $6.2 \mu \mathrm{m}$ band often contains more than one component.

These emission features are indicative of the presence of aromatic hydrocarbons (Duley \& Williams 1981, Leger \& Puget 1984, Allamandola et al. 1989) and other bands, notably those in the 3.4-3.6 $\mu \mathrm{m}$ range, are characteristic of $\mathrm{CH}$ vibrations in $s p^{3}$-hybridized bonded hydrocarbons (Duley \& Williams 1983). However, the composition of the compounds responsible for these bands is still uncertain despite numerous theoretical and laboratory studies (Scott et al. 1997, Hudgins et al. 1999, 2004, van Diedenhoven et al. 2004). This difficulty arises because the dominant observational bands at 6.2 and $7.7 \mu \mathrm{m}$ do not appear at these wavelengths in laboratory or theoretical emission spectra of known PAH molecules. This suggests that the species responsible for the emission bands are either much larger molecules, or are radicals and radical ions derived from fully hydrogenated molecules through dehydrogenation and ionization in interstellar sources.

While the general features of these emission spectra are reproduced in laboratory spectra of hydrogenated amorphous carbon (HAC) (Scott et al. 1997, Grishko \& Duley 2000, Duley et al. 2005), detailed assignments are precluded due to broadening in these solids. This broadening occurs because of the overlap of spectral features from a wide variety of molecular components. We have subsequently found a way to significantly reduce this broadening by using surface enhanced Raman spectroscopy (SERS) to sample highly-localized areas on the surface of carbon nanoparticles. Essentially this technique enables spectra to be obtained from molecular-sized units. An important aspect of SERS is that the technique samples both Raman and infrared-active vibrations (Etchegoin et al. 2003). As a result, spectra represent the full density of states and can therefore be directly compared with those emitted by large carbon molecules in astronomical sources.

\section{Experimental}

Thin films of amorphous carbon $(\mathrm{a}: \mathrm{C})$ and HAC were deposited at temperatures between 77 and $573 \mathrm{~K}$ on clean fused quartz substrates or polished p-type Si wafers with (100) orientation. A base pressure of $2 \times 10^{-7}$ Torr $\left(2.67 \times 10^{-5} \mathrm{~Pa}\right)$ was obtained in a high vacuum chamber evacuated by a turbo-molecular pump with a liquid nitrogen trap. One mJ pulses from a Ti:sapphire laser $(\lambda=800 \mathrm{~nm}$, pulse duration $\tau=120$ fs, repetition frequency $500 \mathrm{~Hz}$, incidence angle $45^{\circ}$ ) were used to ablate high purity pyrolytic graphite (99.99\%). Sample microstructure was characterized with a scanning electron microscope equipped with a field emission gun and with a commercial atomic force microscope. In general, these films were found to be assembled from individual nanoparticles having sizes $<100 \mathrm{~nm}$. Further details on the structure and properties of these materials can be found elsewhere (Hu et al. 2006, 2007). Film thickness was measured using a profilometer (AMBios XP-2) after deposition. The SERS technique requires that the sample be coated with a thin silver layer (Dieringer et al. 2006). To avoid possible contamination with organic solvents, the silver layer in these experiments was deposited in vacuum using laser ablation of Ag metal. Raman spectra were obtained using a Renishaw micro-Raman spectrometer with a He-Ne laser source at an excitation wavelength of $633 \mathrm{~nm}$. The spectral resolution was $1 \mathrm{~cm}^{-1}$. The morphology of these samples is as shown in Figure 1. These materials have a nano-assembled structure whereby carbon nano-particles (CNPs) combine to form a range of larger structures (Hu et al. 2007a, b).

Some advantages of the SERS technique are that it can be used to sample molecularsized units and that the energies of transitions are accurately reproduced for molecules such as naphthalene. In addition, both infrared and Raman transitions appear in SERS 


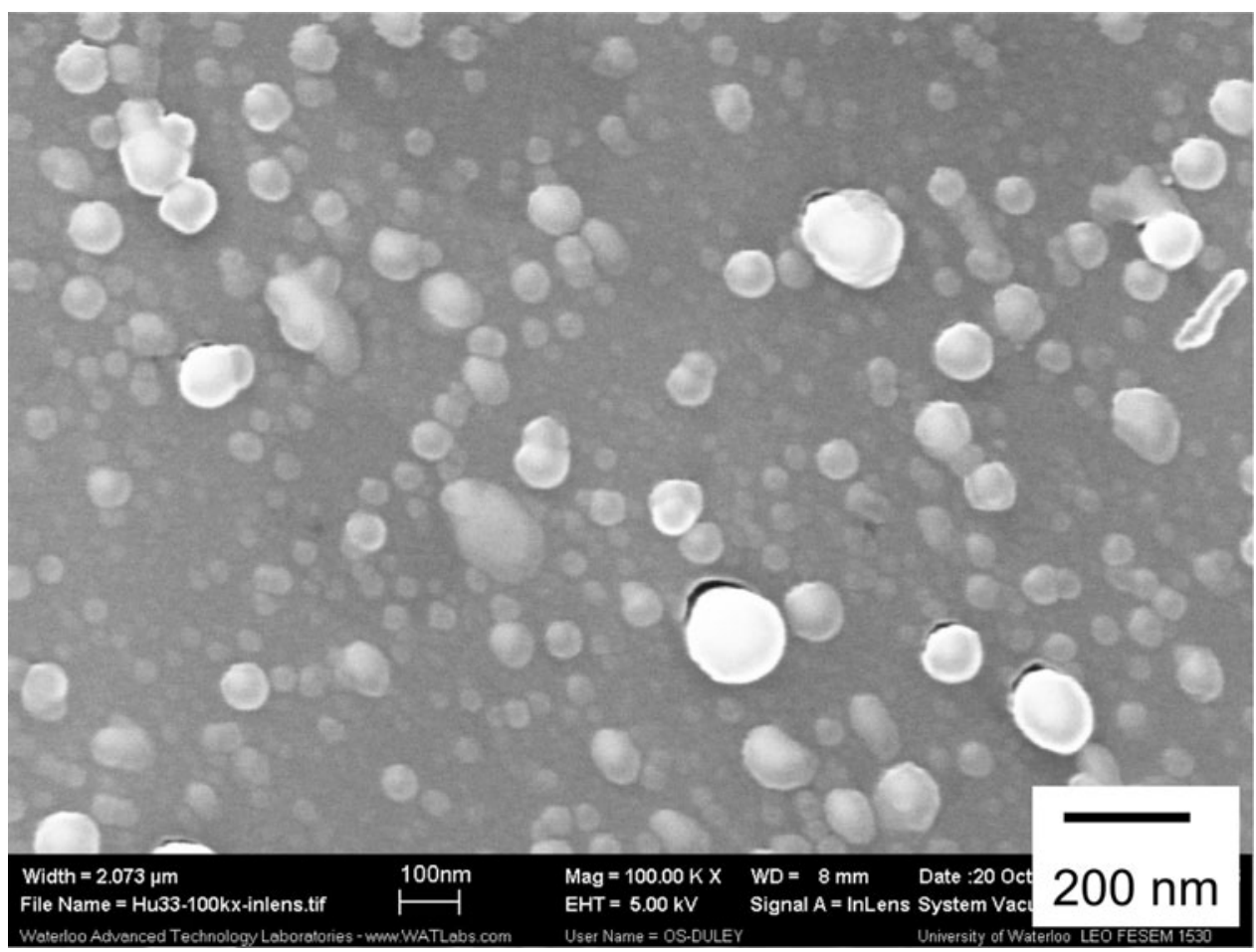

Figure 1. Nano-assembled structure of CNP sample. Individual particles have sizes of several $\mathrm{nm}$. These combine to form larger aggregates.

spectra (Moskovits 1985). The linewidth of these transitions is determined by vibrational delocalization on timescales of $1-10$ psec $\left(10 \mathrm{~cm}^{-1}\right)$, similar to that which occurs in ISM spectra.

\section{Laboratory spectra of CNPs and astronomical spectra}

A summary of several spectral features seen in CNP samples is given in Figure 2. These originate from $\mathrm{CH}$ molecular groups present in individual carbon nanoparticles. Apart from the band at $3.26 \mu \mathrm{m}$, which corresponds to the stretching vibration of aromatic $\mathrm{CH}$, all features have linewidths in the $10 \mathrm{~cm}^{-1}$ range comparable to those observed in interstellar spectra. The larger width of the $3.26 \mu \mathrm{m}$ feature is consistent with rapid delocalization of excitation in the aromatic CH stretching band (Hu \& Duley 2007, 2008). The SERS technique is especially sensitive to the presence of $\mathrm{C}=\mathrm{C}$ bonds, so spectra tend to be enhanced at wavelengths where the vibrations of these species occur. Two low-resolution survey spectra (Figure 3) illustrate this point. These show strong peaks near 1600 and $1320 \mathrm{~cm}^{-1}(6.25$ and $7.6 \mu \mathrm{m})$ that can be identified with vibrational modes of $\mathrm{C}=\mathrm{C}$ bonds in aromatic ring and other hydrocarbon molecules (Ferrari \& Robertson 2000). It is important to note that only the peaks near $7.6 / 7.8 \mu \mathrm{m}$ are diagnostic of the presence of aromatic rings since the $\mathrm{C}=\mathrm{C}$ vibration in other non-aromatic hydrocarbon molecules can also contribute to the $6.2 \mu \mathrm{m}$ band. The features in Figure 3 all contain structure arising from individual molecular groups and are, themselves, superimposed on a broad continuum extending from $\sim 1700-1100 \mathrm{~cm}^{-1}(5.9-9.1 \mu \mathrm{m})$ similar to that commonly observed in astronomical spectra (Allamandola et al. 1989, Peeters et al. 2002). The relative strength of the two main peaks in Figure 3 is found to be variable, but there is no obvious detailed correlation between the relative amplitude of the 6.2 and 
7.6/7.8 $\mu \mathrm{m}$ peaks and preparation conditions. This suggests that both aromatic and non-aromatic molecules contribute to the band near $6.2 \mu \mathrm{m}$. There is also little difference in the wavelengths of these peaks in hydrogenated and de-hydrogenated samples. This result is expected since a range of ring compositions are present in all of the samples studied and ring frequencies in large molecules are little affected by hydrogenation. For example, the spectrum of coronene is strongly correlated with that of graphite (Mapelli et al. 1999). This suggests that the spectra reported here for molecular groups within clusters will be representative of individual PAH species.
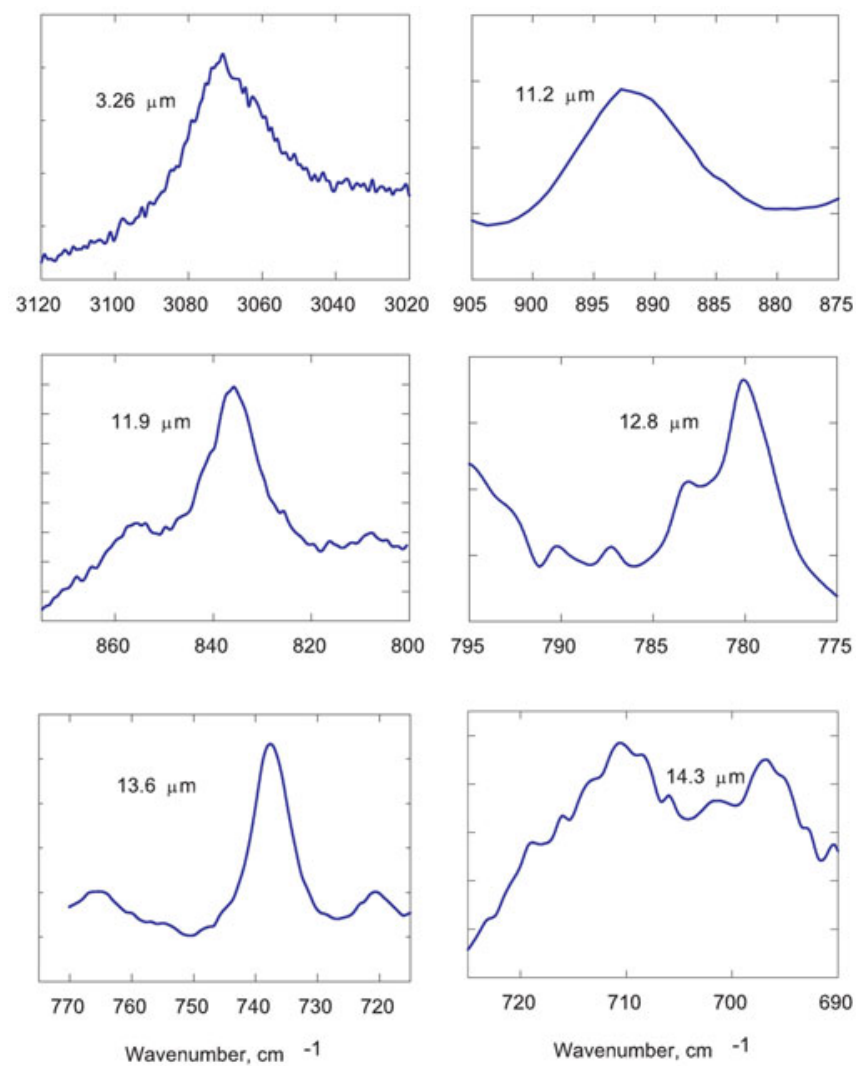

Figure 2. A selection of spectra of CNPs corresponding to the $\mathrm{CH}$ stretching and bending modes in $\mathrm{PAH}$ groups in these particles. The stretching band of $\mathrm{CH}$ appears at 3.26 microns, while other bands correspond to bending modes of $\mathrm{CH}$ in various configurations.

Spectra of three samples representative of A, B and C-type features in the $6.2 \mu \mathrm{m}$ region are shown in Figure 4. Vertical lines show the average wavelength of the observed features in this range. None of these spectra are "pure" in the sense that they all show structure. The appearance of different profiles is then due to variations in the relative strength of these components. For example, the peak of the type C profile in Figure 4 appears as a shoulder in the type B spectrum. Measurements also indicate that the $6.2 \mu \mathrm{m}$ feature extends from 6.06-6.45 $\mu \mathrm{m}\left(1650-1550 \mathrm{~cm}^{-1}\right)$ implying that the observation of type $\mathrm{A}, \mathrm{B}$, or $\mathrm{C}$ emission may simply reflect changes in the excitation and/or concentration of a limited number (4-5) of specific molecular structures. There is also evidence for another component at $5.98 \mu \mathrm{m}\left(1671 \mathrm{~cm}^{-1}\right)$ in the type B spectrum (Figure 4) that could correspond to the weak emission feature observed at $22486.0 \mu \mathrm{m}$ in the spectrum 


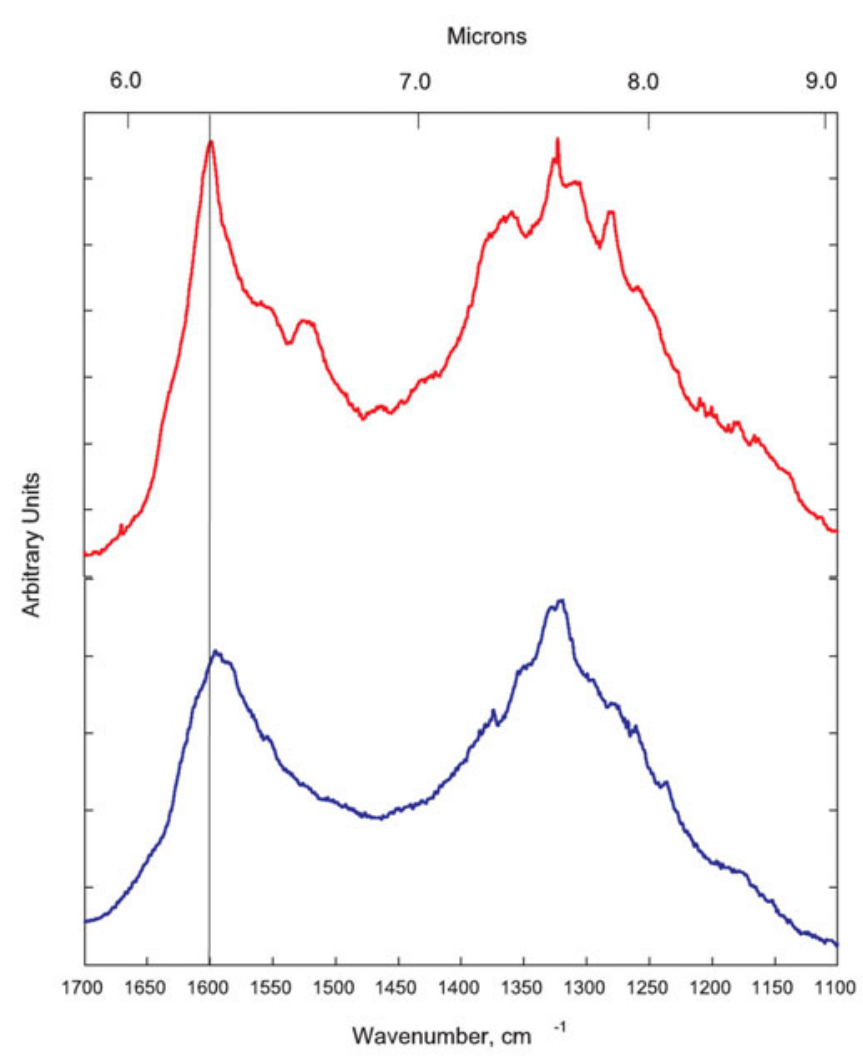

Figure 3. Low-resolution SERS spectra of samples in the $1700-1100 \mathrm{~cm}^{-1}(5.9-9.0 \mu \mathrm{m})$ range. Upper spectrum: nano-assembled film of amorphous carbon $(\mathrm{a}: \mathrm{C})$. Lower spectrum: nano-assembled film of HAC (a-C:H).

of HD44179 and other objects (Peeters et al. 2002). Emission at this wavelength is characteristic of non-aromatic $\mathrm{C}=\mathrm{C}$ groups (Duley 2000), although it could also arise from an overtone and/or combination band involving ring vibrations (Allamandola et al. 1989).

Spectra of several CNPs samples in the region of the $3.3 \mu \mathrm{m}$ aromatic $\mathrm{CH}$ stretching band are shown in Figure 5. All have a well-defined spectral feature that can be associated with the $\mathrm{CH}$ stretching band in PAH molecules. As expected, since these samples are recorded at $300 \mathrm{~K}$, the wavelength of this feature is blue-shifted from the type $\mathrm{A}$ and type B interstellar emission bands. The FWHM of the laboratory feature in Figure 5a is virtually identical to that of the type A emission band $\left(224837 \mathrm{~cm}^{-1}, \sim 0.04 \mu \mathrm{m}\right)$, while that in Figure $5 \mathrm{~b}$ is somewhat narrower $\left(\sim 23 \mathrm{~cm}^{-1}, \sim 0.025 \mu \mathrm{m}\right)$. The wavelength of the aromatic $\mathrm{CH}$ band in our samples ranges between $3.27 \mu \mathrm{m}\left(3057 \mathrm{~cm}^{-1}\right)$ in Figure $5 \mathrm{a}$ to $3.255 \mu \mathrm{m}\left(3072 \mathrm{~cm}^{-1}\right)$ in Figure 5 d. This variation arises because of detailed changes in the molecular composition of individual samples and reflects the fact that CNPs, as prepared in our experiments, are not identical. Nanoscale inhomogeneities in these samples can arise from variations in the size and substitution of PAH components, as well as from inclusion of different amounts of non-aromatic hydrocarbons.

A direct comparison between the profiles of the laboratory and interstellar aromatic $\mathrm{CH}$ bands (Hu \& Duley 2008) yields a good fit to both the type A and type B spectra after shifts of -18 and $-24 \mathrm{~cm}^{-1}(+0.019$ and $+0.026 \mu \mathrm{m})$, respectively, in the laboratory spectra. These shifts are consistent with the higher effective excitation temperature, $T_{\text {ism }}$, of emitters in interstellar sources (Joblin et al. 1995). From the measurements of Joblin 


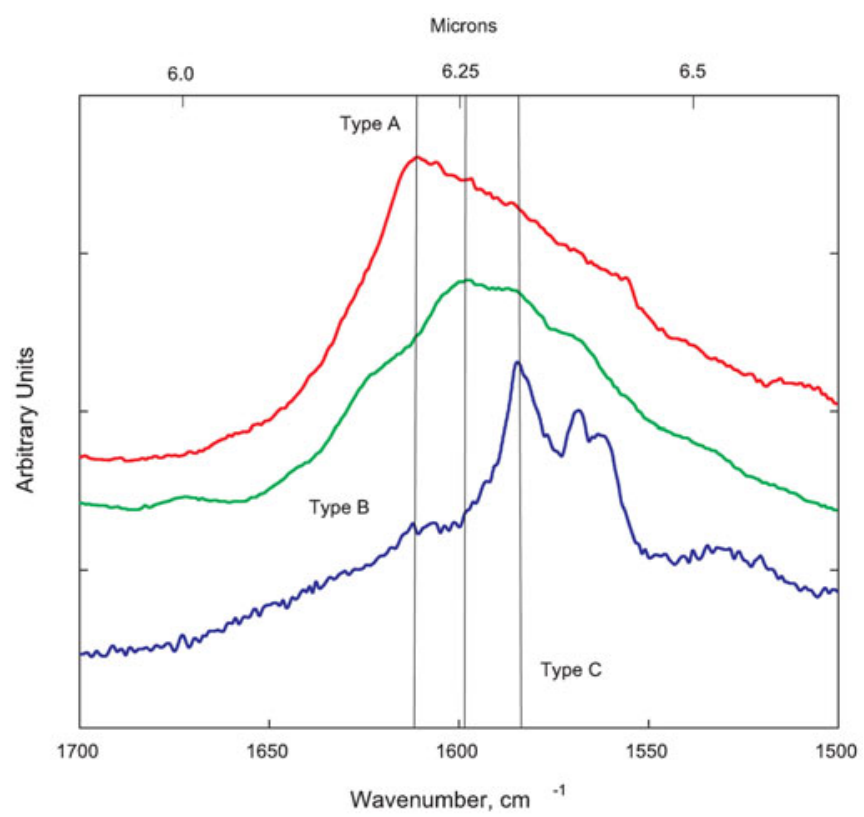

Figure 4. Spectra of three samples exhibiting a range of $6.2 \mu \mathrm{m}$ profiles. Type A, B and C designations are from Peeters et al. (2002).

et al. (1995), the aromatic $\mathrm{CH}$ band is expected to shift by $\sim-0.033\left(T_{\mathrm{ism}}-T_{\mathrm{lab}}\right) \mathrm{cm}^{-1}$. Then with $T_{\text {lab }}=300 \mathrm{~K}$, shifts of -18 and $-24 \mathrm{~cm}^{-1}$ as required to fit the type $\mathrm{A}$ and type B profiles with the laboratory spectrum, correspond to $T_{\mathrm{ism}} \sim 850$ and $1025 \mathrm{~K}$, respectively. These values are in agreement with estimated excitation temperatures for the $3.29 \mu \mathrm{m}$ feature in interstellar sources.

The laboratory experiments of Joblin et al. (1995) predict that the width of the $3.29 \mu \mathrm{m}$ feature should also increase with temperature, but this is not consistent with the fits shown $\mathrm{Hu}$ et al. (2008). This discrepancy likely occurs because the profile of the Raman line is broadened by delocalization (on a timescale of 1 psec) of the excitation centered on the $\mathrm{CH}$ stretching band, rather than as a result of thermal heating. Thus, the width of the Raman feature more closely reflects the processes occurring in the excitation of the interstellar emitters, resulting in a spectral width that differs from that of thermal broadening. In this context, we have previously found that line widths as small as several $\mathrm{cm}^{-1}$ are observed in SERS spectra of CNPs at longer wavelengths (Hu \& Duley 2007, 2008). Narrowing of spectral lines is expected at long wavelengths because of a reduction in the rate of energy de-localization at lower vibrational energies.

Our laboratory data is in agreement with a model in which the type A band is the emission counterpart of the DISM absorption feature at $3.275 \mu \mathrm{m}$ (Hu \& Duley 2008). We reach this conclusion based on the following information: i) SERS spectra of CNPs at $300 \mathrm{~K}$ show an aromatic $\mathrm{CH}$ band at $3.27 \mu \mathrm{m}\left(3057 \mathrm{~cm}^{-1}\right)$ only $-0.0043 \mu \mathrm{m}\left(+4 \mathrm{~cm}^{-1}\right)$ away from the DISM absorption feature, ii) the profile of this feature closely matches that of the type A emission peak, iii) thermal emission spectra of our samples show that the $\mathrm{CH}$ peak occurs at $3.294 \pm 0.002 \mu \mathrm{m}\left(3036 \pm 2 \mathrm{~cm}^{-1}\right)$ at $800 \mathrm{~K}$ (Grishko \& Duley 2000). CNP samples that exhibit type A bands in our spectra also contain an aliphatic component in the form of chains bonded to PAH structures (Hu et al. 2007b), suggesting that PAHs in the DISM likely have aliphatic side-chains. A model in which $\mathrm{CH}$ groups on PAHs are replaced by aliphatic side-chains in diffuse clouds is consistent 


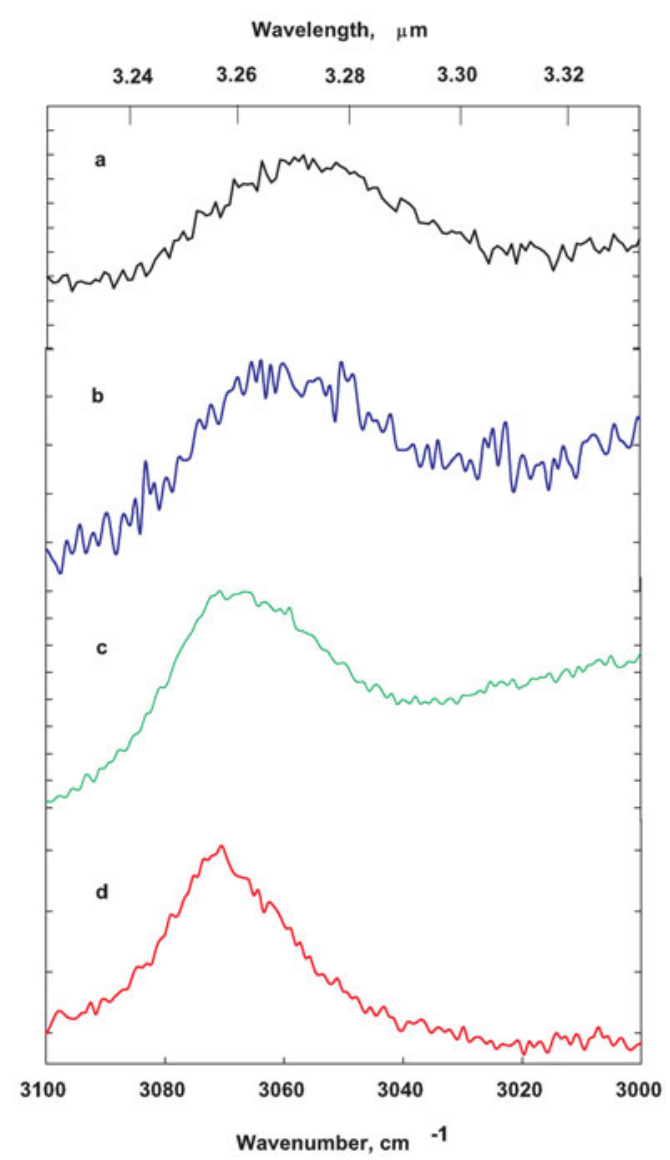

Figure 5. SERS spectra in the $3.29 \mu \mathrm{m}\left(3040 \mathrm{~cm}^{-1}\right)$ range of CNP samples at $300 \mathrm{~K}$. Individual peaks correspond to excitation of the $\mathrm{CH}$ stretching mode in aromatic (PAH) molecular groups. Other data suggests that the average size of the PAH structures in these samples decreases from spectrum (a) to spectrum (d). PAHs in sample (d) may contain as few as one or two C6 rings.

with the observation that the $3.4 \mu \mathrm{m}$ absorption band from $\mathrm{CH}_{2}$ and $\mathrm{CH}_{3}$ groups is much stronger than the aromatic CH band in DISM material (Dartois et al. 2007) and that the $3.29 \mu \mathrm{m} \mathrm{CH}$ emission feature is very weak under these conditions (Rouan et al. 1999).

It is notable that a broad absorption feature, likely attributable to another type of $\mathrm{PAH}$, is observed at $3.255 \mu \mathrm{m}\left(3072 \mathrm{~cm}^{-1}\right)$ in spectra of molecular clouds (Sellgren 2001). We see a feature at this wavelength in many of our CNP samples containing small rings (Figure $5 \mathrm{c}-\mathrm{d}$ ) and associate it with benzene, naphthalene and anthracene molecules having aliphatic side-chains. It is possible that small PAH molecules of this type are only present in these heavily obscured objects where they are shielded from UV radiation.

\section{Conclusions}

Laboratory spectra of CNPs reproduce many of the infrared emission features, including their spectral widths. The agreement between the specral linewidths observed in SERS spectra and that seen in interstellar emission occurs because the Raman process results in similar internal vibrational energy excitation. Spectral shifts required to fit the emission peak of the $3.29 \mu \mathrm{m}$ band is consistent with vibrational heating to $\sim 800$ $1,000 \mathrm{~K}$. This suggests that the DISM absorption band at $3.275 \mu \mathrm{m}$ arises from the same chemical species as that which produces type A emission in many astronomical sources. 
These emitters are indicated to be relatively small (2-7 ring) PAH molecules with one or more attached aliphatic side-chains.

This research was supported by a grant from the NSERC of Canada. The author thanks Anming $\mathrm{Hu}$ for providing data and Q. B. Lu for the use of laser facilities.

\section{References}

Allamandola, L. J., Tielens, A. G. G. M., \& Barker, J. R. 1989, ApJS, 71, 733

Dartois, E. 2007, A\& A, 463, 635

Dieringer, J. A. 2006, Faraday Disc., 132, 9

Duley, W. W. 2000, ApJ, 528, 841

Duley, W. W. \& Williams, D. A. 1981, MNRAS, 196, 269

Duley, W. W. \& Williams, D. A. 1983, MNRAS, 205, 67P

Duley, W. W., et al. 2005, ApJ, 626, 923

Etchegoin, P., et al. 2003, Chem. Phys. Lett., 367, 223

Ferrari, A. C. \& Robertson, J. 2000, Phys. Rev. B, 61, 14095

Geballe, T. R. 1997, in: Y. J. Pendleton \& A. G. G. M. Tielens (eds.), From Stardust to Planetisimals, ASP Conf. Ser. 122, (San Francisco:ASP), p. 109

Grishko, V. \& Duley, W. W. 2000, ApJ (Letters), 543, L85

Hu, A., Alkhesho, I., Duley, W. W., \& Zhou, H. 2006, J. Appl. Phys., 100, 084319

Hu, A., Alkhesho, I., Zhou, H., \& Duley, W. W. 2007a, Diamond and Related Materials, 16, 149 Hu, A. \& Duley, W. W. 2007, ApJ, 672, L81

Hu, A. \& Duley, W. W. 2008, ApJ, in press

Hu, A., Lu, Q-B., Duley, W. W., \& Rybachuk, M. 2007b, J. Chem. Phys., 126, 154705

Hudgins, D. M. \& Allamandola, L. J. 1999, 513, L69

Hudgins, D. M. \& Allamandola, L. J. 2004, in: A. N. Witt, G. C. Clayton, \& B. T. Draine (eds.), ASP Conf. Ser. 309, Astrophysics of Dust, (San Francisco:ASP), p. 665

Joblin, C., Boissel, P., Leger, A., d'Hendecourt, L., \& Defourneau, D. 1995, A\&̋A, 299, 835,

Leger, A. \& Puget, J. L. 1984, A\&A (Letters), 137, L5

Mapelli, C., Castiglione, C., Zerbi, G. \& Mullen, K. 1999, Phys. Rev. B, 60, 12710

Moskovits, M. 1985, Rev. Mod. Phys., 57, 78

Peeters, E., et al. 2002, A\&GA, 390, 1089

Peeters, E., Mattioda, A. L., Hudgins, D. M. \& Allamandola, L. J. 2004, ApJ (Letters), 617, L65

Rouan, D., Le Coupanec, P., Lacombe, F., Tiphene, D., Gallais, P., Leger, A., \& Boulanger, F. 1999, in The Universe as seen by ISO, ESA, SP-427, p. 743

Scott, A., Duley, W. W., \& Jahani, H. R. 1997, ApJ (Letters), 490, L175

Sellgren, K. 2001, Spectrochimica Acta, A57, 627

Sloan, G. C., et al. 2005, ApJ, 632, 956

Tokunaga, A. T. 1996, in: H. Onaka, T. Matsumoto \& T. L. Roelig (eds.), Diffuse Infrared Radiation and the IRTS, ASP Conf. Ser. 124, (San Francisco:ASP), p. 149

van Diedenhoven et al., 2004, ApJ, 611, 928

Werner, M. W., et al. 2004, ApJS, 154, 309

\section{Discussion}

Mulas: You compared your spectrum of the $3.3 \mu \mathrm{m}$ band with the interstellar one, applied the shift due to temperature, and showed that you still have a small residual. I'd note that in the interstellar medium you will also have overtones, and they will be exactly where you have the residual.

Duley: Yes, that's the possibility.

Mulas: Also, in one of your slides you showed the absorption of the $3.3 \mu \mathrm{m}$ aromatic band in a molecular cloud. Could an alternative explanation be simply that, in that case, you have a molecule that is at very low temperature, so you see the reverse of this shift? 
DULEY: It's too much of a shift. It has to be a different species.

HenNING: These are very nice data in the infrared. Do you have any absorption spectra for the optical or UV to constrain the absorption spectrum?

Duley: I have a new student who's going to do a lot of work in these other regions.

Smith: Have you taken a look at the $3.4 \mu \mathrm{m}$ feature?

DulEy: Yes, we have a lot of data on that, but we haven't quite sorted out what is going on. There are a few surprises there that I think will be quite interesting. We've been working our way through the spectrum, and taking as it comes ...

SARRE: One of the key observational aspects is the strength of bands in the 5 to $6 \mu \mathrm{m}$ region. Can the laboratory data explain why the $\mathrm{C}-\mathrm{C}$ modes are strong in some regions, and not in others?

Duley: Well, for the C-C modes it depends on the wavelength. They can shift, and if they are not rings, they can really shift quite a bit. Keep in mind that you can have C-C modes but not have C-H modes if you don't have a high degree of hydrogenation. If you have a large particle that just has a lot of rings, then the C-C modes can be quite strong, and the other bands that are associated with hydrogen are really reduced. You can vary that ratio by changing the level of hydrogenation in the molecule.

SARRE: Is that really seen in the lab?

Duley: Yes, we do see variations in composition. The composition depends on the gas in which samples are prepared.

ZiURYs: Did you ever do experiments where you add a little bit of oxygen or nitrogen to produce heteroatom structure?

DULEY: Yes, but it's not actually that easy to get these heteroatoms in there, particularly the nitrogen. We've tried, and we've got some data, but it's not something I am comfortable with. They don't seem to like to get into these molecules under our experimental conditions. There may be a channel that produces this in some special way, but so far we haven't really found it.

ZiURYs: How do you ablate your graphite? Do you use a graphite rod?

DUlEY: We use highly oriented pyrolytic graphite. It's basically layers of graphene.

ZIURYS: Did you ever think of drilling a hole though it and running in oxygen and nitrogen?

Duley: Yes, but it doesn't work well. It will oxidize around where you are heating, but it doesn't get into the product very easily. 


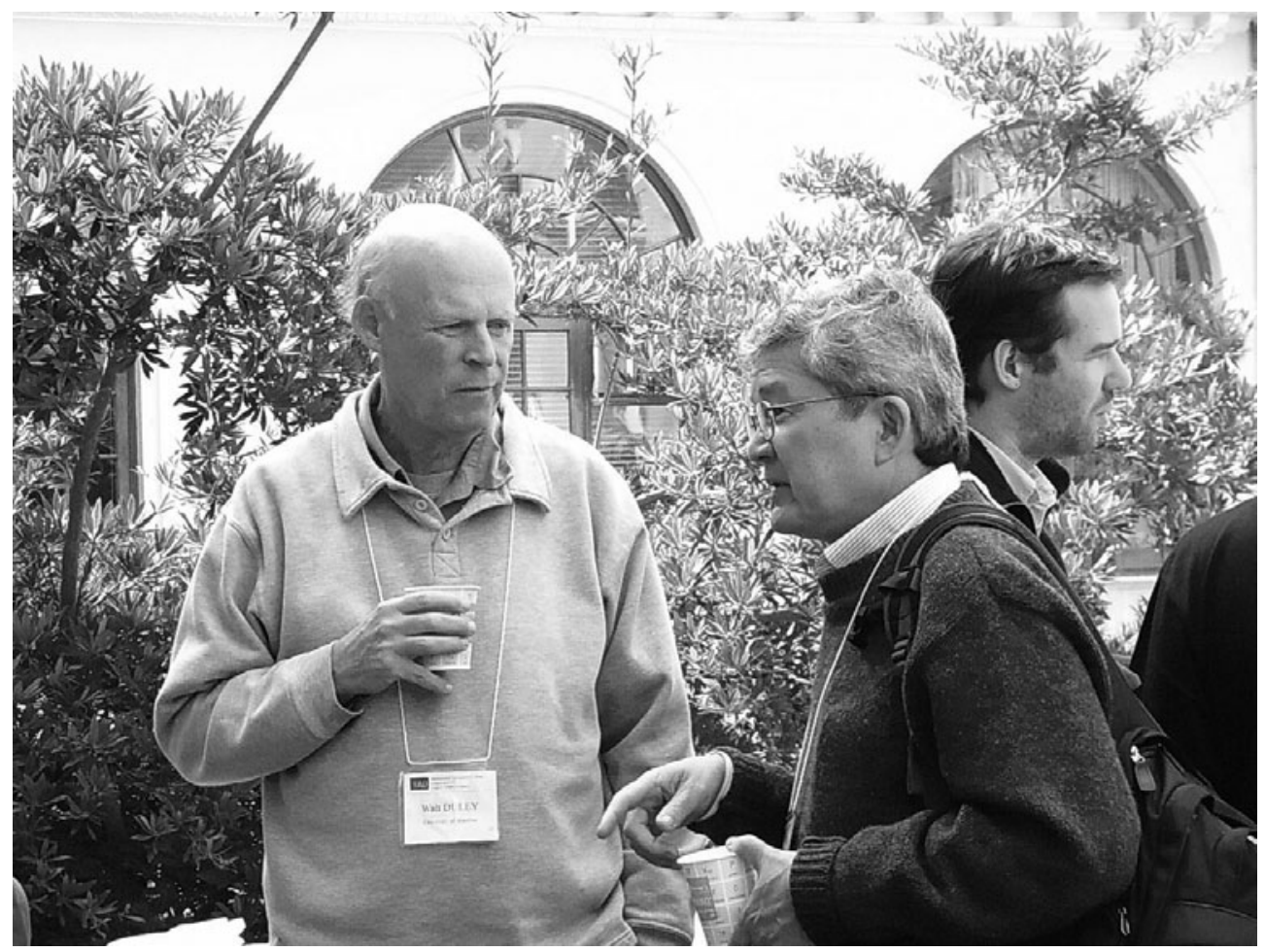

Walt Duley (left) and Alan Tokunaga at coffee. 